\title{
Developing a Motion Language: Gait Analysis from Accelerometer Sensor Systems
}

\author{
Anita Sant'Anna \\ School of Informations Sciences, \\ Electrical and Computer Engineering \\ Halmstad University - Sweden \\ Email: Anita.Santanna@hh.se
}

\author{
Nicholas Wickström \\ School of Informations Sciences, \\ Electrical and Computer Engineering \\ Halmstad University - Sweden \\ Email: Nicholas.Wickstrom@hh.se
}

\begin{abstract}
This work concerns the use of human movement classification as a tool for monitoring and supporting older peoples' lives. The "motion language" methodology is a movement classification technique which aims at generalizing movements and providing easy interpretation of motion signals by decomposing activities into elementary building blocks referred to as "motion primitives". The use of motion primitives to classify motion from visual data has been studied. This work shows that the motion language methodology can be applied to acceleration signals, contributing to the development of wearable monitoring systems. This paper explains the development of the motion language and its use in a gait analysis study. Preliminary results show that the motion language methodology can be used to quantitatively measure gait parameters. In addition, motion primitives are shown to express static and dynamic characteristics of different gait patterns and were used to calculate a new symmetry index.
\end{abstract}

\section{INTRODUCTION}

The world's population is expected to age considerably in the next 50 years [1]. Projections show that by 2050 the older population (aged 65 or over) will grow from $16 \%$ to $28 \%$ of the total population of Europe (Table I). This change in demographics is mostly due to the increase in life-expectancy and the aging of the Post-World-War-II baby boomers. Today's health care system will struggle to cope with the increased demand of age related care in the coming years.

Table I

PROJECTIONS OF THE PERCENTAGE OF THE POPULATION AGED 65 OR OVER IN DIFFERENT REGIONS.

\begin{tabular}{|c|c|c|c|}
\hline Year & $\mathbf{2 0 0 5}$ & $\mathbf{2 0 3 0}$ & $\mathbf{2 0 5 0}$ \\
\hline World & $7.3 \%$ & $11.7 \%$ & $16.2 \%$ \\
\hline Europe & $15.9 \%$ & $22.6 \%$ & $27.6 \%$ \\
\hline Sweden & $17.2 \%$ & $22.8 \%$ & $24.1 \%$ \\
\hline UK & $16.1 \%$ & $21.6 \%$ & $24.1 \%$ \\
\hline US & $12.3 \%$ & $19.4 \%$ & $21.0 \%$ \\
\hline Japan & $19.7 \%$ & $2 \%$ & $24.1 \%$ \\
\hline
\end{tabular}

Some ways of relieving the health care system from the extra load are: to focus on preventive care, to support aging at home by ensuring safety and assistance in activities of daily living, and to aim at early diagnosis of common conditions. The health and safety of a senior at home may be monitored by intelligent systems which share relevant information with medical personnel and informal care-takers, such as family members and friends. Movement analysis is a powerful and intuitive way of determining a subject's health and functional status [2]. Gait analysis, in particular, provides relevant information about physical and cognitive decline [3].

Many studies have focused on movement recognition for evaluating balance, e.g. [4], and classifying gait, e.g. [5]. Such studies, however, are based on template matching techniques and they have been useful for detecting only a few predetermined activities or events. Another approach to movement classification is to decompose activities into small building blocks referred to as "motion primitives". These units may be organized as a motion language, which is able to express different activities as a combination of primitives.

This work investigates the application of the motion language methodology to acceleration data in order to extract gait parameters and describe dynamically different walking patterns through a measure of symmetry. A new symmetry index $\left(S I_{s y m b}\right)$ is proposed and compared to a traditional measure of symmetry $(S I)$. The symmetry index proposed here is shown to be more informative, and its interpretation more intuitive, than the traditional symmetry index.

This paper is organized as follows: related work is discussed in Section II; the motion language methodology is explained in Section III; the experimental setup is presented in Section IV; the design of the motion language is explained in Section V; the extraction of gait characteristics based on motion primitives is presented in Section VI; results are analyzed in Section VII; future work is discussed in VIII; and Section IX concludes this paper.

\section{RELATED WORK}

\section{A. Gait Analysis}

Gait analysis is an important tool for the diagnosis and evaluation of many conditions. Previous studies have shown that changes in gait can be related to physical and cognitive decline [6] due to aging or illness [7]. Common causes of gait abnormalities in senior citizens include neurological diseases, arthritis and acquired foot deformities [8]. Stroke survivors also frequently display gait abnormalities [9].

Gait analysis has been evaluated for predicting the risk of developing dementia and, in particular, the risk of developing 
Alzheimer's disease [8]. Another study [10] has quantified that a 1-second increase in time to walk 30ft, when compared to control subjects, can be indicative of an increased risk of developing permanent cognitive impairment. The correlation between slowing of gait and mild cognitive impairment is also supported in [11]. In addition, gait analysis may help identify and quantify the risk of an elder falling [12], and it is an essential tool in the treatment and evaluation of cerebral palsy patients [13], [14]. Gait analysis is also important when fitting prosthetic or orthotic devices, and when evaluating the success of an orthopedic surgery, e.g. a hip-replacement [15], [16].

Gait analysis is already widely used for clinical assessment, but it is often subjective or constrained to a laboratory environment. Motion capture (mocap) systems, in combination with force-plates, provide very accurate descriptions and models of gait. However, these expensive systems must be installed in appropriate rooms and can only be operated by specially trained personnel. Mocap systems can only record movements performed in a small area of the room. Logging long walks is therefore only possible if the patient is walking on a treadmill, which may change the patient's normal gait. Other measuring systems used in the lab setting, more mobile but less informative, are pressure sensitive mats [17]. Pressure mats provide static spacio-temporal data, analogous to footprints over time. They do not, however, provide dynamic information about how the patient's foot is moving in space.

When measuring systems are unavailable, gait and balance are commonly assessed using "time up and go" tests (TUG) and/or grading scales [18], [19]. However, studies have shown that these tests can be subjective and sometimes inconsistent, especially when the tester is inexperienced [20]. Another drawback of current approaches to clinical gait assessment is that tasks performed in the lab or clinic do not always simulate normal daily activities. Consequently, such measurements may fail to reflect the subject's normal walking pattern. There is, therefore, a clear need for an inexpensive, unobtrusive and easy-to-use system, which allows quantitative analysis of gait patterns outside the lab. In this scenario, wearable sensor systems composed of inertial sensors such as accelerometers and gyros [21] are particularly useful. Wearable sensor systems certainly overcome the mobility issue, and they are preferable to visual data, since people may feel uncomfortable being recorded by camera-like devices.

\section{B. Wearable Sensor Systems}

A large number of sensors is inconvenient for most longterm monitoring applications. In addition, when designing wearable systems, a clear trade off is observed between mobility and ease of use in terms of: power consumption, autonomy, placement, patient compliance, and data analysis, to name a few. Therefore, one approach to developing new portable sensor nodes is to start with only one type of sensing device and investigate how much information it can gather.

Accelerometers are suitable sensors for wearable systems since current enhancements in micro-electro-mechanical systems (MEMS) technology have made possible the manufacture of miniaturized, low power, low cost accelerometers [21], which are useful for logging human motion data for long periods of time in uncontrolled environments. Accelerometers have been employed for many different purposes: estimating metabolic energy expenditure [22]; monitoring activity [20]; assessing standing balance [4]; detecting falls, postural orientation and classifying activities [2].

Gait analysis studies from accelerometer sensor systems may be divided into three main categories:

1) The reconstruction of movements in space, e.g. [23], [24]. These studies make use of two or more sensors on the same limb in order to reconstruct its trajectory in space and, as such, are not comparable to our work.

2) The detection of gait phases and evaluation of temporal parameters, e.g. [25]-[27]. These studies focus on detecting events such as heel-strike or stance and use this information to calculate stride times, variability, temporal symmetry, etc. They do not, however, provide any information about the way in which the person is walking, i.e if the person's feet are moving according to different trajectories in space.

3) The classification of walking patterns, e.g. [5], [28]. These studies aim at determining if the subject is walking up or down stairs, running etc. They do not, on the other hand, look into single steps and the phases of gait.

Complementing previous studies, the motion language methodology proposed in this work aims at both tasks: identifying the phases of gait, and describing the dynamics of the walking pattern.

\section{Motion LANGUage}

A fundamental problem in detecting and recognizing human movements is that of representation. Movement classification has traditionally been achieved through some form of template matching or pattern recognition, after manual segmentation and/or statistical feature modeling. These methods require manual labeling of the data, which is labor-intensive and errorprone [29].

The motion language methodology aims at identifying an "alphabet" of elementary motions, which are similar to building blocks for human movements [30]. This motion alphabet enables the creation of a "motion language", where analogies are made between movements and words. The relationship between action and language is supported by the Mirror Neuron Theory [31] which states that the same brain mechanisms are activated regardless of whether actions are being performed or observed [32].

A motion language is able to generalize movement by describing innumerable concepts using different combinations of a limited number of primitives. The organization of elementary actions for classifying human movement by describing a hierarchical model has already been studied [33]. The concept of motion primitives was also explored in a method which automatically derived vocabularies of movement modules from visual data by taking advantage of the underlying spatialtemporal structure of human movements [29]. Video image 
sequences have been converted into strings containing sequences of symbols, each representing a manually determined primitive, to classify five one-arm movements [34]. Also from video images (mocap database), the inference of sequential and parallel grammar rules to describe human movements has been studied [35]. The motion-primitive concept has also been used for motor modular control of robots, frequently combined with learning through observation [36], [37].

Based on previous approaches, the development of a motion language may be divided into four general tasks: Segmentation, Feature Extraction, Symbolization and Grammar Inference. Together, these four tasks constitute the motion language methodology.

\section{A. Segmentation}

One of the challenges in the process is determining how to segment the signal into suitable building blocks. Most motion language approaches, specially those based on fullbody mocap data, manually segment motion into primitives, e.g. movements decomposed into individual joints' angular displacement. When the data available is of reduced dimensionality, and not so intuitive as visual data, the signal should be automatically segmented, i.e. without human input, according to its inherent characteristics in order to maximize the amount of information the symbols retain from the original signal.

\section{B. Feature Extraction}

After segmentation, various features extracted from each segment of the signal are used to classify them into different symbols. The most common feature extraction approach, when detecting daily activities from accelerometer data, is to extract information from equally sized sliding windows. This does not take primitives into consideration but rather statistical information about the whole movement.

\section{Symbol Assignment}

The features extracted from the segments are used to differentiate them from one another. Segments with similar characteristics may be assigned one symbol. Finding appropriate features is important to the creation of relevant symbols.

\section{Grammar Inference}

Grammar rules express how symbols may be put together to form words and sentences which represent different movements. They may be inferred from a large collection of data or designed based on known characteristics of the system, e.g. the human locomotive system. Although segmentation, feature extraction, and symbol assignment may not relate directly to movements, the physical limitations of the body should be reflected by grammar rules on syntactic and semantic levels. Syntactic analysis is concerned with deriving rules about which movements are possible, such as "arms only bend one way". Semantic analysis considers rules about how different movements are associated. Humans cannot, for example, "chew gum and whistle at the same time". The elaboration of grammar rules from mocap data has been explored in [35].

\section{EXPERIMENTAL SETUP}

Two SHIMMER sensor nodes (shimmer-research.com), composed of tri-axial accelerometers, were attached to both shins of the subjects, close to the ankles. The placement of the sensor was chosen so that the user would be able to strap the sensors on without much precision. The movements were sampled at $50 \mathrm{~Hz}$ and the data was continuously streamed via Bluetooth to a nearby computer. The subjects walked a straight line on a six-meter-long Gold Gait Rite pressure mat [17], which samples data at $60 \mathrm{~Hz}$. The data obtained from the pressure mat was used as a reference for heel-strike and toe-off.

Seven subjects participated in the experiments. They were asked to walk: (1) at a comfortable self-paced speed, referred to throughout this paper as normal walk; (2) at a very slow speed taking shorter steps, slow walk; and (3) at a comfortable self-paced speed while having their right knee immobilized with a brace in order to simulate limping, limp walk. Three runs of each type of walk were performed on the 6-meterlong pressure mat. The number of steps recorded varied from around 10 , for normal and limp walking, to around 30 , for slow walking. Only steps with good reference data were considered. The data was analyzed and the results obtained from one subject's data are presented in Section VII.

\section{Motion Language Design}

The goal of this study was to apply the proposed motion language methodology to acceleration data in order to extract gait parameters and symmetry measures for the three different types of walk describe in Section IV. A new measure of symmetry was derived from the motion primitives and compared with a traditional symmetry index. Throughout this paper, Stance is defined as the period when the foot is on the ground; swing is when the foot is off the ground; toe-off is the moment when the foot leaves the ground and heel-strike is the moment when the foot meets the ground. Stride is the complete sequence: heel-strike, stance, toe-off, swing and heel-strike.

The acceleration signals used in this study were not calibrated to $m / s^{2}$ hence the acceleration units will be omitted. In longer data collection sessions, sensors might exhibit drift, in which case some form of adaptive calibration would be required.

The motion language methodology was implemented as follows:

\section{A. Segmentation}

The accelerometer data was filtered with a mean filter three samples wide. The Segmentation task was performed on the resultant acceleration signal:

$$
A_{\text {res }}=\sqrt{A_{x}^{2}+A_{y}^{2}+A_{z}^{2}},
$$

where $A_{\text {res }}$ is the resultant acceleration, and $A_{x}, A_{y}, A_{z}$ are accelerations in the accelerometer's local coordinate system. When the subject is standing still, with both feet together, $x$ corresponds to the vertical axis, $y$ corresponds to the 
horizontal axis perpendicular to the direction of walking, and $z$ corresponds to the horizontal axis along the direction of walking.

The chosen segmentation method was a bottom-up linear segmentation algorithm described in [38]. The algorithm starts by fitting a small line segment over every consecutive three samples and iteratively fits longer line segments over neighboring samples until the mean square error (MSE) between the original resultant acceleration and the linearly segmented signal reaches a predetermined error threshold. The data points between start and end of each fitted line are regarded as a segment.

The output of the Segmentation task is illustrated in Figure 1. The diamond-shaped markers indicate the beginning and the end of each segment. The dotted line represents the resultant acceleration before segmentation and the solid line represents the segmented signal.

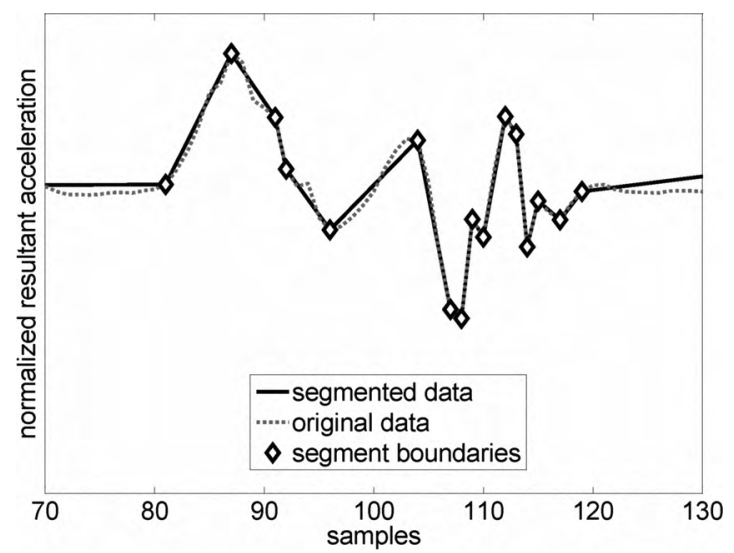

Figure 1. Segmented signal compared to original resultant acceleration signal, for "normal walk".

\section{B. Feature Extraction}

The features extracted from each segment were: variance of the resultant acceleration, var; mean acceleration on all three axes, $\overline{A_{x}}, \overline{A_{y}}, \overline{A_{z}}$; and the number of samples in the segment, length. These features were chosen since they represent well the data in each segment (mean and variance) and its duration (length). The $i$ th segment is then represented by the fivedimensional vector:

$$
\mathbf{S}_{\text {feat }}(i)=\left[\overline{A_{x}}(i) \overline{A_{y}}(i) \overline{A_{z}}(i) \operatorname{var}(i) \text { length }(i)\right]
$$

\section{Symbol Assignment}

The segment features extracted from the acceleration signal for both the right, $\mathbf{S}_{\text {featR }}$, and the left foot, $\mathbf{S}_{\text {feat } L}$, were normalized to the interval [0 1]. Considering $N$ segments from the right foot and $M$ segments from the left foot, the data is organized in a $(N+M) \times 4$ matrix:

$$
\left(\begin{array}{c}
\mathbf{s}_{\text {feat } R}(1) \\
\mathbf{s}_{\text {feat } R}(2) \\
\vdots \\
\mathbf{s}_{\text {feat } R}(N) \\
\mathbf{S}_{\text {feat } L}(1) \\
\mathbf{s}_{\text {feat } L}(2) \\
\vdots \\
\mathbf{s}_{\text {featL }}(M)
\end{array}\right)
$$

The rows of the matrix above (segments) are divided into different groups using k-means clustering. The optimum number of clusters was chosen based on the minimum Davies-Bouldin index [39]. A different symbol was assigned to the segments within each cluster. For simplicity, the symbols are integers contained in the interval between 1 and the maximum number of clusters.

At this stage, it is not known how or if the symbols are semantically significant. However, it is expected that the acceleration peaks observed at heel-strike and toe-off [40] are characterized by at least one symbol. It is the goal of the "grammar inference" phase to determine how these symbols are associated with the different gait phases. Considering that $\mathrm{K}$-means clustering is sensitive to initial conditions, it could happen that the symbols are not semantically significant and the grammar inference phase fails to identify appropriate rules. In that case, the clustering phase is repeated with different initial conditions.

\section{Grammar Inference}

The rules describing the symbol sequences were manually derived based on the repetitiveness of the signal and the characteristics of the system, e.g. the symbols considered interesting appear once every cycle, toe-off can only come between stance and swing, etc. The rules were used to identify four gait parameters: heel-strike, toe-off, swing and stance. Although only two parameters are needed to determine the other two, i.e. either toe-off and heel-strike or stance and swing, all four parameters were estimated individually so recurrent rules could be designed to maximize the accuracy of the classification. The rules were chosen to be subject and gait type specific due to large interpersonal variability. In the case of normal walking, for one subject, for example, the correspondence between symbols and gait parameters was designed as shown in Table II.

Table II

EXAMPLE OF GRAMMAR RULES

\begin{tabular}{|l|l|}
\hline symbolic occurrence & gait parameter \\
\hline \hline symbol 5 & swing \\
\hline symbol 3 & stance \\
\hline $\begin{array}{l}\text { transition from symbol 4 to symbol 1 between swing } \\
\text { and stance }\end{array}$ & heel-strike \\
\hline $\begin{array}{l}\text { transition from symbol 2 to symbol 4 between stance } \\
\text { and swing }\end{array}$ & toe-off \\
\hline
\end{tabular}


An algorithm may be designed to find these rules automatically by analyzing every symbol and transition, and matching those to known characteristics of the system, e.g. a complete stride is a sequence of heel-strike, swing, toe-off and stance; swing is approximately $40 \%$ of the total stride time; one foot can only swing while the other is standing, etc.

\section{Extracting Gait Characteristics}

As mentioned at the beginning of Section $\mathrm{V}$, the goal of this study is to make use of motion primitives in order to detect the four main phases of gait, and identify different walking patterns.

Heel-strikes identified with the previously mentioned grammar rules, and heel-strikes obtained from the reference signal, were used to calculate stride times. Stride times were, in turn, used to calculate gait symmetry, $S I$, according to (1) [41]. This gait measurement is important when assessing, e.g. rehabilitation after stroke.

$$
S I=\frac{T_{R}-T_{L}}{\frac{1}{2}\left(T_{R}+T_{L}\right)} 100
$$

where $T_{R}$ is the average stride time for the right foot and $T_{L}$ is the average stride time for the left foot.

The closer the absolute value of $S I$ is to zero, the more symmetric the walk. A negative value indicates that the left foot is, on average, slower than the right foot and a positive value indicates the opposite. A slower stride time, however, does not indicate a more abnormal movement and the "affected" side cannot be determined. The value for this index is unbounded and, in practice, a correspondence between this index and quality of gait is unclear. This measure of symmetry only takes into account the average stride time for each foot, and as such, it does not provide any information about the different movements performed by both feet. If the subject limps but manages to walk with similar stride times, the $S I$ index will not consider this to be an asymmetric walk.

The derived symbols can be used to compute a more informative measure of symmetry which takes all the acceleration data into account, as follows. After substituting the acceleration signal for the respective symbol sequence, histograms of the time elapsed between two consecutive symbol transitions of the same kind are calculated for all possible transitions, i.e. $\{1$ to 1,1 to 2,1 to $3, \ldots, N$ to $N\}$, where $N$ is the number of symbols (see Figure 2). The symmetry index based on the transition histograms, $S I_{\text {symb }}$, is computed by:

$$
S I_{s y m b}=\frac{\sum_{i, j=1}^{N} \frac{1}{n_{i j}} \sum_{k=1}^{K}\left|h_{R i j}(k)-h_{L i j}(k)\right|}{\sum_{i, j=1}^{N} \frac{1}{n_{i j}} \sum_{k=1}^{K}\left|h_{R i j}(k)+h_{L i j}(k)\right|},
$$

where $N$ is the number of symbols; $K$ is the number of bins in the histograms; $n_{i j}$ is the number of non-empty histogram bins for transition $i j$, i.e. from symbol $i$ to symbol $j ; h_{R i j}(k)$ is the normalized value for bin $k$ in the transition histogram $i j$ for the right foot; and $h_{L i j}(k)$ is the normalized value for bin $k$ in the transition histogram $i j$ for the left foot.

This index ranges from 0 to 1 , where 0 means total symmetry and 1 means complete asymmetry. $S I_{\text {symb }}$ takes

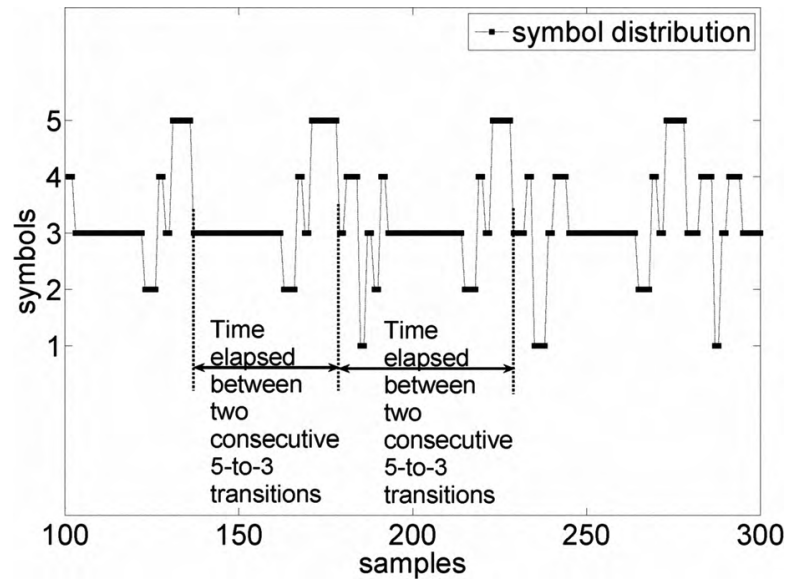

Figure 2. Time elapsed between two consecutive transitions of the same kind (5-to-3). Values found for all such transitions are used to construct transition histograms.

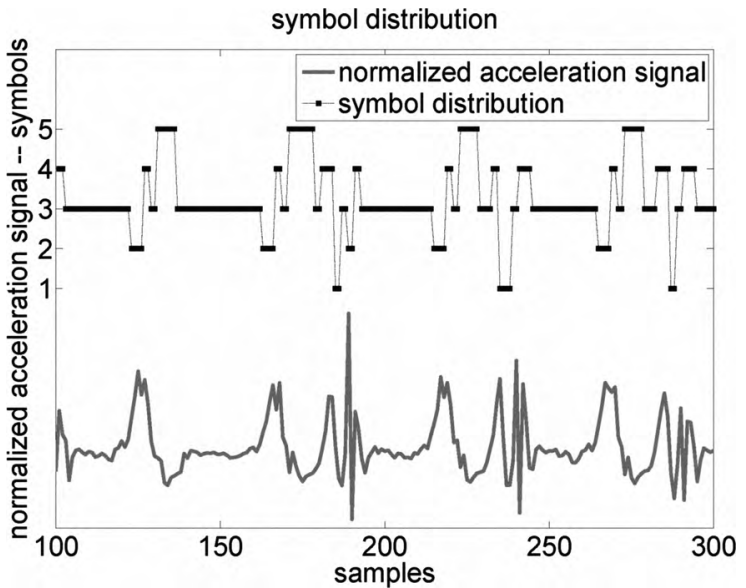

Figure 3. Symbol distribution over time compared to the original resultant acceleration data, for "normal walk".

into account not only the stride times but also the dynamics of the movement. The $S I_{\text {symb }}$ index demonstrates one way in which the symbol abstraction used in the motion language methodology can be used to extract meaningful information from sensor data.

\section{ANALYSIS OF RESULTS}

Figure 3 illustrates the symbols found for the normal walk data (right foot) along with the resultant acceleration, and Figure 4 shows the same symbols along with the reference data from the pressure mat. The symbols found for the limp walk data are shown in Figure 5.

The symbols found for "normal walk" and "slow walk" data were very similar and the rules describing gait events were the same for both feet. On the other hand, the symbols found for "limp walk" were very different from one foot to the other. The different symbols, in this case, reflect the asymmetric nature 

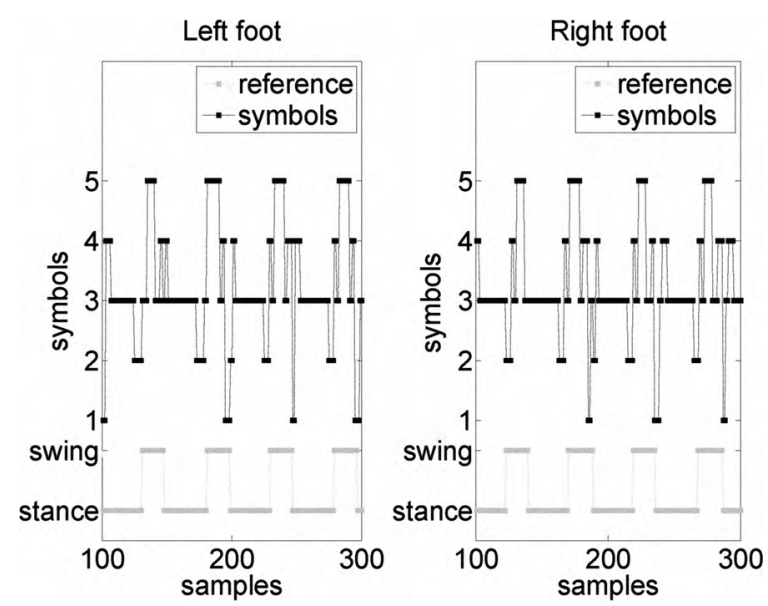

Figure 4. Symbol distribution over time compared to the reference signal from pressure sensitive mat, for "normal walk". Symbolic representation and reference data are similar between both feet.
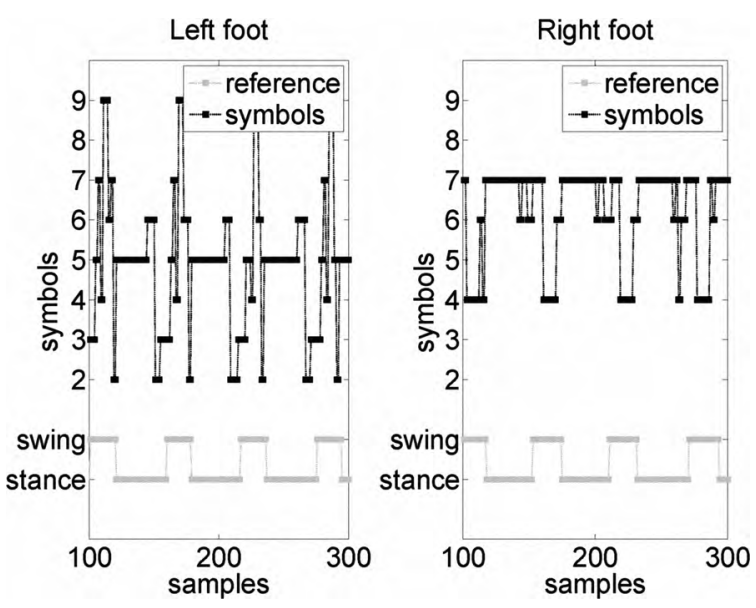

Figure 5. Symbol distribution over time compared to the reference from the pressure sensitive mat, for "limp walk". Reference data looks similar between both feet, but the symbolic representation expresses differences in the movements of each foot.

Table III

NUMBER OF CLUSTERS FOUND IN SYMBOL ASSIGNMENT FOR EACH TYPE OF WALK.

\begin{tabular}{|c|c|c|c|}
\hline $\begin{array}{c}\text { Type of } \\
\text { walk }\end{array}$ & Normal & Slow & Limping \\
\hline $\begin{array}{c}\text { no. of } \\
\text { clusters }\end{array}$ & 5 & 3 & 9 \\
\hline
\end{tabular}

of the walk. In addition, the difference in movement between feet increased the complexity of the data and more symbols were needed to describe the walk. The number of clusters found during the Symbol Assignment task, for this particular subject, are shown in Table III.

Figure 6 illustrates the parameters identified using the proposed motion language methodology, shown alongside the reference signal. The detection of heel-strike and toe-off for

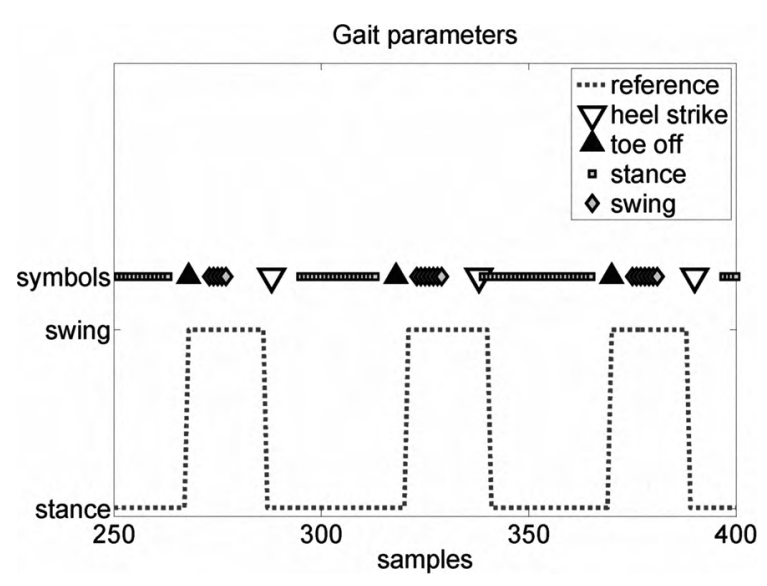

Figure 6. Gait parameters determined from grammar rules after segmentation, feature extraction, and symbolization, for "normal walk".
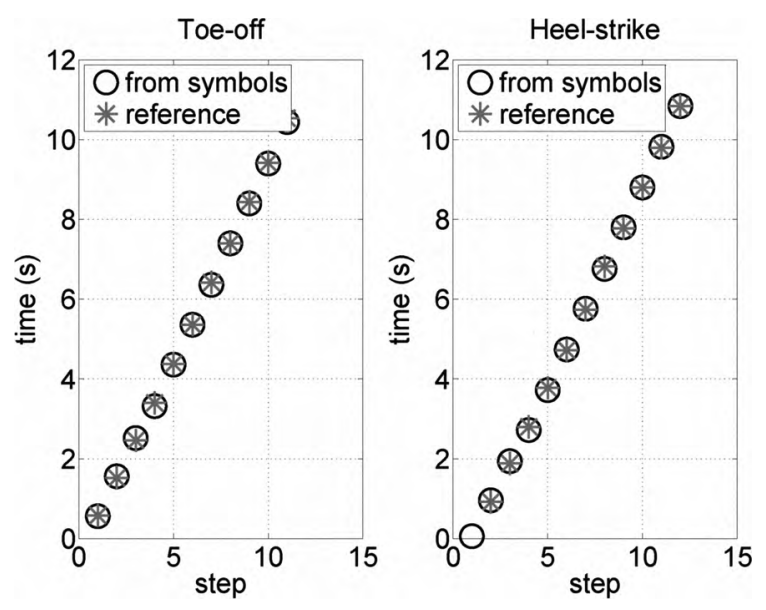

Figure 7. Heel-strike and toe-off detection compared to the reference signal from the pressure sensitive mat, for "normal walk".

the right foot from the normal walk data set are displayed in Figure 7.

The average heel-strike and toe-off detection errors are very small for true positives (see Tables IV and V). The false positives and false negatives happened, in general, at the start or end of the data set, where there were no symbols before or after to be inferred from (conditional rules). Future work might avoid this by using longer data sets. Pressure sensitive insoles may be more appropriate than the pressure mat for data collection, since they may provide continuous reference for heel-strike and toe-off for longer periods of time.

The $S I$ and $S I_{\text {symb }}$ symmetry indices for all the three types of walk are shown in Table VI. The same one-second difference in average stride time between feet, for slow walk and limp walk, resulted in different absolute values of $S I$. The $S I$ index is biased by the stride time. The $S I$ symmetry index found for the limp walk, both from the reference data and from the symbols, is close to zero. This value does not express the asymmetry of the limping pattern. Though the subject was 
Table IV

OVERVIEW OF THE RESULTS FOR HEEL-STRIKE DETECTION.

\begin{tabular}{|c|ll|ll|ll|}
\hline \multirow{2}{*}{ Type of Walk } & \multicolumn{2}{|c|}{ Normal } & \multicolumn{2}{c|}{ Slow } & \multicolumn{2}{c|}{ Limping } \\
\cline { 2 - 8 } & $\begin{array}{l}\text { Left } \\
\text { Foot }\end{array}$ & $\begin{array}{l}\text { Right } \\
\text { Foot }\end{array}$ & $\begin{array}{l}\text { Left } \\
\text { Foot }\end{array}$ & $\begin{array}{l}\text { Right } \\
\text { Foot }\end{array}$ & $\begin{array}{l}\text { Left } \\
\text { Foot }\end{array}$ & $\begin{array}{l}\text { Right } \\
\text { Foot }\end{array}$ \\
\hline no. of Steps & 9 & 11 & 30 & 29 & 12 & 9 \\
\hline $\begin{array}{c}\text { no. of False } \\
\text { Negatives }\end{array}$ & 0 & 0 & 1 & 2 & 1 & 1 \\
\hline $\begin{array}{c}\text { no. of False } \\
\text { Positives }\end{array}$ & 0 & 1 & 2 & 1 & 1 & 0 \\
\hline $\begin{array}{c}\text { Average Error of } \\
\text { True Positives (s) }\end{array}$ & 0.03 & 0.04 & 0.07 & 0.04 & 0.02 & 0.03 \\
\hline
\end{tabular}

Table V

OVERVIEW OF THE RESULTS FOR TOE-OFF DETECTION.

\begin{tabular}{|c|ll|ll|ll|}
\hline \multirow{2}{*}{ Type of Walk } & \multicolumn{2}{|c|}{ Normal } & \multicolumn{2}{c|}{ Slow } & \multicolumn{2}{c|}{ Limping } \\
\cline { 2 - 8 } & $\begin{array}{l}\text { Left } \\
\text { Foot }\end{array}$ & $\begin{array}{l}\text { Right } \\
\text { Foot }\end{array}$ & $\begin{array}{l}\text { Left } \\
\text { Foot }\end{array}$ & $\begin{array}{l}\text { Right } \\
\text { Foot }\end{array}$ & $\begin{array}{l}\text { Left } \\
\text { Foot }\end{array}$ & $\begin{array}{c}\text { Right } \\
\text { Foot }\end{array}$ \\
\hline no. of Steps & 9 & 11 & 30 & 29 & 12 & 9 \\
\hline $\begin{array}{c}\text { no. of False } \\
\text { Negatives }\end{array}$ & 0 & 0 & 1 & 0 & 0 & 0 \\
\hline $\begin{array}{c}\text { no. of False } \\
\text { Positives }\end{array}$ & 0 & 0 & 1 & 2 & 0 & 0 \\
\hline $\begin{array}{c}\text { Average Error of } \\
\text { True Positives (s) }\end{array}$ & 0.03 & 0.04 & 0.07 & 0.06 & 0.03 & 0.02 \\
\hline
\end{tabular}

Table VI

OVERVIEW OF THE RESULTS FOR SYMMETRY INDICES, $S I$ AND $S I_{s y m b}$.

\begin{tabular}{|c|c|c|c|c|c|c|}
\hline \multirow{2}{*}{ Type of Walk } & \multicolumn{2}{|c|}{ Normal } & \multicolumn{2}{|c|}{ Slow } & \multicolumn{2}{|c|}{ Limping } \\
\hline & $\begin{array}{l}\text { Left } \\
\text { Foot }\end{array}$ & $\begin{array}{l}\text { Right } \\
\text { Foot }\end{array}$ & $\begin{array}{l}\text { Left } \\
\text { Foot }\end{array}$ & $\begin{array}{l}\text { Right } \\
\text { Foot }\end{array}$ & $\begin{array}{l}\text { Left } \\
\text { Foot }\end{array}$ & $\begin{array}{l}\text { Right } \\
\text { Foot }\end{array}$ \\
\hline $\begin{array}{c}\text { Average stride } \\
\text { time (s) } \\
\text { (symbols) }\end{array}$ & 0.98 & 0.98 & 1.29 & 1.28 & 1.17 & 1.18 \\
\hline $\begin{array}{c}\text { Average stride } \\
\text { time (s) } \\
\text { (reference) }\end{array}$ & 0.98 & 0.98 & 1.29 & 1.28 & 1.17 & 1.17 \\
\hline$S I$ (symbols) & \multicolumn{2}{|c|}{0} & \multicolumn{2}{|c|}{-0.78} & \multicolumn{2}{|c|}{0.85} \\
\hline$S I$ (reference) & \multicolumn{2}{|c|}{0} & \multicolumn{2}{|c|}{-0.78} & \multicolumn{2}{|c|}{0} \\
\hline$S I_{\text {symb }}$ & \multicolumn{2}{|c|}{0.12} & \multicolumn{2}{|c|}{0.04} & \multicolumn{2}{|c|}{0.95} \\
\hline
\end{tabular}

moving each leg differently, the average stride time for both feet was still similar. The $S I_{\text {symb }}$ symmetry index, however, picks up on the asymmetry of the limping gait. According to the $S I_{\text {symb }}$ index, limping is less symmetric than normal or slow walking, and its value is not biased by the stride time.

\section{FUTURE WORK}

This study has laid the ground work for a number of further investigations. A more extensive analysis of the data is still to come, which will compare symbols across subjects and attempt to find correspondences between the symbols found for each type of walk. The goal of this next study will be to find symbols robust enough to describe different subjects' gait patterns, and comprehensive enough to span different walking patterns, rendering the analysis subject independent. In addition, an algorithm will be designed to automatically extract rules describing gait parameters, based on previous knowledge of the system (unsupervised learning).

Three possible paths to finding more informative symbols seem particularly interesting to consider: investigating new segmentation methods; investigating new features and rules; and investigating new clustering and symbolization techniques. New segmentation methods could be based on statistical characteristics of the signal, such as the probability of certain values occurring, or non-linear local descriptions, such as reoccurring sequences of values. New features and rules could exploit the parallelism between feet. In normal walking patterns, for example, both feet should be performing the same activity but shifted in time. Using this information may improve or ease the detection of gait parameters. The third path may investigate appropriate initial conditions for the clustering method or the use of system identification approaches and hybrid Markov models to cluster and symbolize the data.

The motion language methodology was here applied to acceleration data, but it can be extended to any time-series. Different types of sensors could undergo the same analysis. This method provides also a good compression of the original signal and can be used to reduce the computational complexity of different techniques in learning or adaptive systems.

\section{CONCLUSION}

The motion language methodology is a powerful tool for representing human movements and it has been used in different research areas. An important application in the health care area is gait analysis. Since accelerometers are inexpensive and convenient sensors for recording human movements, this work investigated the used of motion primitives to analyze accelerometer-based gait data.

Results show that the motion language methodology can be used for identifying gait parameters such as heel-strike and toe-off, as well as gait symmetry. The symmetry index based on motion primitives proposed here outperforms the traditional index in that it is not biased and it conveys not only static but also dynamic information about the gait pattern. This symmetry index is an example of how the motion language can contribute to the understanding of movements, the interpretation of accelerometer signals, and therefore, the development of wearable monitoring systems.

\section{ACKNOWLEDGMENT}

The authors would like to thank: Dr. Misha Pavel and Dr. Holly Jimison, for making the data collection possible; Dr. Fay Horak, for welcoming us into her lab; and Dr. Arash Salarian, for technical support.

\section{REFERENCES}

[1] U. Nations, World Population Prospects: The 2006 RevisionComprehensive Tables. United Nations Publications, 2007. 
[2] D. M. Karantonis, M. R. Narayanan, M. Mathie, N. H. Lovell, and B. G. Celler, "Implementation of a real-time human movement classifier using a triaxial accelerometer for ambulatory monitoring," Information Technology in Biomedicine, IEEE Transactions on, vol. 10, no. 1, pp. 156-167, 2006.

[3] R. Camicioli, D. Howieson, B. Oken, G. Sexton, and J. Kaye, "Motor slowing precedes cognitive impairment in the oldest old," Neurology, vol. 50 , no. 5 , p. $1496,1998$.

[4] R. Mayagoitia, J. Lötters, P. Veltink, and H. Hermens, "Standing balance evaluation using a triaxial accelerometer," Gait \& Posture, vol. 16, no. 1 , pp. 55-59, 2002.

[5] R. K. Ibrahim, E. Ambikairajah, B. G. Celler, and N. H. Lovell, "Timefrequency based features for classification of walking patterns," Digital Signal Processing, 2007 15th International Conference on, pp. 187-190, 2007.

[6] A. Kluger, J. Gianutsos, J. Golomb, A. Wagner, D. Wagner, and S. Scheurich, "Clinical features of MCI: motor changes," International Psychogeriatrics, vol. 20, no. 01, pp. 32-39, 2007.

[7] S. Frenkel-Toledo, N. Giladi, C. Peretz, T. Herman, L. Gruendlinger, and J. Hausdorff, "Effect of gait speed on gait rhythmicity in Parkinson's disease: variability of stride time and swing time respond differently," Journal of NeuroEngineering and Rehabilitation, vol. 2, no. 1, p. 23, 2005.

[8] J. Verghese, R. Lipton, C. Hall, G. Kuslansky, M. Katz, and H. Buschke, "Abnormality of Gait as a Predictor of Non-Alzheimer's Dementia," New England Journal of Medicine, vol. 347, no. 22, p. 1761, 2002.

[9] H. Cruz et al., "Evidence of Abnormal Lower-Limb Torque Coupling After Stroke: An Isometric Study* Supplemental Materials and Methods," Stroke, vol. 39, no. 1, p. 139, 2008.

[10] S. Marquis, M. Moore, D. Howieson, G. Sexton, H. Payami, J. Kaye, and R. Camicioli, "Independent Predictors of Cognitive Decline in Healthy Elderly Persons," Archives of Neurology, vol. 59, no. 4, pp. 601-606, 2002.

[11] R. Roberts, J. Hollman, Y. Geda, D. Knopman, R. Cha, V. Pankratz, W. Rocca, and R. Petersen, "P4-118: Impairment in gait is associated with prevalent mild cognitive impairment," Alzheimer's \& Dementia: The Journal of the Alzheimer's Association, vol. 4, no. 4S, pp. 704 704, 2008.

[12] C. Toulotte, A. Thevenon, E. Watelain, and C. Fabre, "Identification of healthy elderly fallers and non-fallers by gait analysis under dual-task conditions," Clinical Rehabilitation, vol. 20, no. 3, p. 269, 2006.

[13] J. R. Gage, "Gait analysis: an essential tool in the treatment of cerebral palsy: Applied kinesiology," Clinical orthopaedics and related research, no. 288, pp. 126-134, 1993.

[14] K. Desloovere, G. Molenaers, H. Feys, C. Huenaerts, B. Callewaert, and P. Walle, "Do dynamic and static clinical measurements correlate with gait analysis parameters in children with cerebral palsy," Gait \& Posture, vol. 24, no. 3, pp. 302-13, 2006.

[15] U. Lindemann, C. Becker, R. Muche, K. Aminian, H. Dejnabadi, T. Nikolaus, W. Puhl, K. Huch, and K. Dreinhofer, "Gait analysis and WOMAC are complementary in assessing functional outcome in total hip replacement," Clinical Rehabilitation, vol. 20, no. 5, p. 413, 2006.

[16] K. Aminian, C. Trevisan, B. Najafi, H. Dejnabadi, C. Frigo, E. Pavan, A. Telonio, F. Cerati, E. Marinoni, P. Robert, et al., "Evaluation of an ambulatory system for gait analysis in hip osteoarthritis and after total hip replacement," Gait \& Posture, vol. 20, no. 1, pp. 102-107, 2004.

[17] A. McDonough, M. Batavia, F. Chen, S. Kwon, and J. Ziai, "The validity and reliability of the GAITRite system's measurements: A preliminary evaluation," Archives of Physical Medicine and Rehabilitation, vol. 82, no. 3, pp. 419-425, 2001

[18] M. Thomas, J. Jankovic, M. Suteerawattananon, S. Wankadia, K. Caroline, K. Vuong, and E. Protas, "Clinical gait and balance scale (GABS): validation and utilization," Journal of the Neurological Sciences, vol. 217, no. 1, pp. 89-99, 2004.

[19] T. Steffen, T. Hacker, and L. Mollinger, "Age-and Gender-Related Test Performance in Community-Dwelling Elderly People: Six-Minute Walk Test, Berg Balance Scale, Timed Up \& Go Test, and Gait Speeds," Physical Therapy, vol. 82, no. 2, p. 128, 2002.

[20] K. Culhane, M. O'Connor, D. Lyons, and G. Lyons, "Accelerometers in rehabilitation medicine for older adults," Age and Ageing, vol. 34, no. 6 , pp. 556-560, 2005.

[21] R. Mayagoitia, A. Nene, and P. Veltink, "Accelerometer and rate gyroscope measurement of kinematics: an inexpensive alternative to optical motion analysis systems," Journal of Biomechanics, vol. 35, no. 4, pp. 537-542, 2002.

[22] C. Bouten, K. Westerterp, M. Verduin, and J. Janssen, "Assessment of energy expenditure for physical activity using a triaxial accelerometer.," Med Sci Sports Exerc, vol. 26, no. 12, pp. 1516-23, 1994.

[23] D. Giansanti, V. Macellari, G. Maccioni, and A. Cappozzo, "Is it feasible to reconstruct body segment 3-D position and orientation using accelerometric data?," Biomedical Engineering, IEEE Transactions on, vol. 50 , no. 4 , pp. 476-483, 2003

[24] K. Liu, T. Liu, K. Shibata, Y. Inoue, and R. Zheng, "Novel approach for lower limb segment orientation in gait analysis using triaxial accelerometers," IEEE/ASME International Conference on Advanced Intelligent Mechatronics, AIM, pp. 488 - 492, 2008.

[25] R. W. Selles, M. A. G. Formanoy, J. B. J. Bussmann, P. J. Janssens, and H. J. Stam, "Automated estimation of initial and terminal contact timing using accelerometers; development and validation in transtibial amputees and controls," IEEE Transactions on Neural Systems and Rehabilitation Engineering, vol. 13, no. 1, pp. 81 - 88, 2005.

[26] T. Liu, Y. Inoue, K. Shibata, and X. Tang, "A wearable inertial sensor system for human motion analysis," Proceedings of IEEE International Symposium on Computational Intelligence in Robotics and Automation, CIRA, pp. $409-413,2005$.

[27] J.-A. Lee, S.-H. Cho, J.-W. Lee, K.-H. Lee, and H.-K. Yang, "Wearable accelerometer system for measuring the temporal parameters of gait," 29th Annual International Conference of IEEE-EMBS, Engineering in Medicine and Biology Society, EMBC'07, pp. 483 - 486, 2007.

[28] M. Sekine, T. Tamura, T. Fujimoto, and Y. Fukui, "Classification of walking pattern using acceleration waveform in elderly people," in Engineering in Medicine and Biology Society, 2000. Proceedings of the 22nd Annual International Conference of the IEEE, vol. 2, 2000.

[29] O. C. Jenkins and M. J. Mataric, "Automated derivation of behavior vocabularies for autonomous humanoid motion," in AAMAS '03: Proceedings of the second international joint conference on Autonomous agents and multiagent systems, pp. 225-232, 2003.

[30] D. D. Vecchio, R. M. Murray, and P. Perona, "Decomposition of human motion into dynamics based primitives with application to drawing tasks," Automatica, vol. 39, pp. 2085-2098, 2003.

[31] C. Holden, "The Origin of Speech," Science, vol. 303, no. 5662 pp. 1316-1319, 2004.

[32] G. Rizzolatti and L. Craighero, "The mirror-neuron system," Annual Review of Neuroscience, vol. 27, no. 1, pp. 169-192, 2004

[33] K. Kahol, P. Tripathi, and S. Panchanathan, "Documenting motion sequences with a personalized annotation system," Multimedia, IEEE, vol. 13, pp. 37- 5, Jan.-March 2006.

[34] P. Fihl, M. B. Holte, T. B. Moeslund, and L. Reng, "Action recognition using motion primitives and probabilistic edit distance," in $A M D O$, pp. 375-384, 2006.

[35] G. Guerra-Filho and Y. Aloimonos, "A language for human action," Computer, vol. 40, no. 5, pp. 42-51, 2007.

[36] S. Schaal, J. Peters, J. Nakanishi, and A. Ijspeert, "Learning movement primitives," Springer Tracts in Advanced Robotics, pp. 561-572, 2004.

[37] S. Nakaoka, A. Nakazawa, K. Yokoi, and K. Ikeuchi, "Leg motion primitives for a dancing humanoid robot," IEEE International Conference on Robotics and Automation, vol. 1, pp. 610-615, 2004.

[38] E. Keogh, "Data Mining in Time Series Databases Tutorial," in Proceedings of the IEEE Int. Conference on Data Mining, 2004.

[39] S. Petrovic, "A Comparison Between the Silhouette Index and the Davies-Bouldin Index in Labelling IDS Clusters," in Proceedings of the 11th Nordic Workshop of Secure IT Systems, pp. 53-64, 2006.

[40] K. Aminian, K. Rezakhanlou, E. De Andres, C. Fritsch, P. F. Leyvraz, and P. Robert, "Temporal feature estimation during walking using miniature accelerometers: an analysis of gait improvement after hip arthroplasty," Medical and Biological Engineering and Computing, vol. 37, pp. 686-691, 1999.

[41] L. Nolan, A. Wit, K. Dudziñski, A. Lees, M. Lake, and M. Wychowañski, "Adjustments in gait symmetry with walking speed in trans-femoral and trans-tibial amputees," Gait \& Posture, vol. 17, no. 2, pp. 142-151, 2003. 